\title{
A design of receiver front-end low-noise electrical tuning amplifier circuit
}

\author{
Xiubin $\mathrm{YE}^{1, \mathrm{a}}$, Di ZHANG ${ }^{2, b}$, Guopeng KOU ${ }^{3, \mathrm{c}}$ \\ ${ }^{1}$ Xiamen University Tan Kah Kee College, Zhangzhou, China \\ ${ }^{2}$ Xiamen University Tan Kah Kee College, Zhangzhou, China \\ ${ }^{3}$ Xiamen University Tan Kah Kee College, Zhangzhou, China

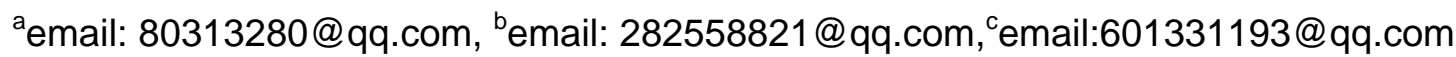

Keywords: Preselector, Filter, Electric Tuning

\begin{abstract}
This paper proposes a design scheme on the low-noise electrical tuning amplifier circuit of receiver front-end. According to key indicators to be achieved, it made a overall simulation design of the front-end amplifier by ADS, focusing on the analysis of the characteristics and parameter selection method of the filter circuit. Finally the circuit is lapped and debugged, its indicators is tested by scanner, compared to the ideal targets, the article analyzed and discussed the error.
\end{abstract}

\section{Introduction}

A variety of electronic equipment used in modern civil and military facilities, resulting in a complex electromagnetic environment and serious interference. Various communication transceivers are generally integrated, the high level interference signals and their intermodulation components generated in the receiver will seriously affect the output SNR of the receiver. In order to reduce the effect, the receiver has the characteristics of high selectivity and high linearity. Therefore, this paper designed a amplifying circuit whose band-pass filter is $400-440 \mathrm{MHz}$, used electric tuning to adjust the bandwidth of the passband. The main principle is that by adjusting a voltage of the variable capacitance diode, the passband always shift follow the center frequency, so we can achieve good reception with about half of the band-pass. Due to the reduction of bandwidth, the noise can be suppress, obtained circuit will have a better performance.

\section{Basic components of the receiver}

The common RF front-end of the microwave repeater is mainly composed of a preselector, a high frequency amplifier, a mirror frequency filter, a mixer, a filter, local oscillator, pre-amplifier e.g., as shown in figure 1 , this paper mainly studied the first three parts of the operating circuit.

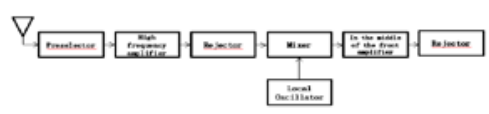

\section{A design of preselector}

Fig.1. RF front-end block

Signals received by receiver antenna has a wide band from low to high frequency. The bandwidth is proportional to the noise power, so noise interference will be worse if amplification without the selection filter. The front-end preselector is a low pass filter with about $500 \mathrm{MHz}$ cutoff frequency and a electronic tuning band pass filter with $40 \mathrm{MHz}$ bandwidth, laying the foundation for the later high frequency amplifier and frequency selective filter. This design uses seven order Butterworth low pass filter, the band pass filter have basically the same principle with the mirror filter.

Implementation indicators:

First: Gain As $>=20 \mathrm{db}$

Second: The cut-off frequency of Fp $=440 \mathrm{MHz}$ 
Third: The characteristic frequency Fs $=500 \mathrm{MHz}$

Fourth: The impedance match $=50 \Omega$ (because the characteristic impedance of the antenna is $50 \Omega$, and the low pass filter front end directly connected to the antenna, so the output impedance of the filter should reach $50 \Omega$ )

The circuit principle diagram as shown in figure 2. We simulate the circuit by ADS, the result as shown in figure 3 and figure 4 :

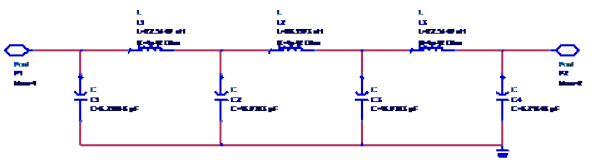

Fig.2. The front-end preselector circuit diagram

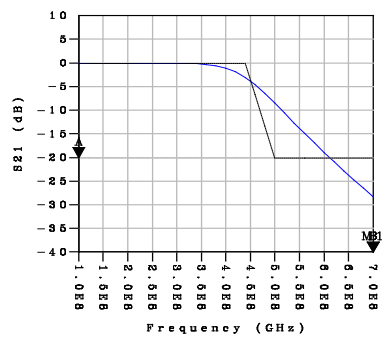

Fig.3. The amplitude frequency



Fig.4. The phase frequency characteristic curve

Measuring by the actual circuit, the total gain is about $25 \mathrm{~dB}$, which is greater than the required (20 dB), the cutting-off frequency is about $450 \mathrm{MHz}$, which meet the basic requirement. Therefore, the design of low pass filter meet the requirements. In the actual circuit, the low-pass effect is better when the subsequent circuit is not loaded. However, duo to the interference of the subsequent circuit, after the low-pass cut-off frequency is in the later, there are some bigger ripple, and the waveform not still decline steeply enough.

\section{A design of a high frequency amplifier}

The performance index is:

First: The power gain Pg>=15dbm amplifier.

Second: The SNR S/N $>=40 \mathrm{db}$ amplifier.

Third: The circuit should have good anti blocking effect, avoid the large signal into the amplifier, it will made the amplifier into the saturated state and lose the amplification effect.

Fourth: The elimination of the self-excited oscillation in the negative feedback.

Fifth: The design of the amplifier must have the very good impedance matching effect to made the triode achieve the best amplification state[1][2]. (Because the input impedance of the common-emitter amplifier is small, but its output impedance is great, at the high frequency, the attention must be paid to the problem of impedance matching, otherwise the signal distortion is very serious.)

The amplification circuits of high frequency small signal is divided into a narrow band amplifying circuit and a wide band amplifier circuit. The former amplifies without distortion the weak signal whose center frequency is from hundreds $\mathrm{kHz}$ to $\mathrm{MHz}$, spectrum width is from the several $\mathrm{kHz}$ to tens $\mathrm{MHz}$. So it not only needs to have a certain voltage gain, but also needs to have the selective ability. The latter amplifies without distortion the weak signal whose band is wider from several $\mathrm{MHz}$ to hundreds $\mathrm{MHz}$. So it requires the lower cut-off frequency very low (some requirements reach the zero frequency mean DC), the upper cut-off frequency very high. This paper adopts the narrow-band amplification and use the triode amplification [3]. The amplification circuit as shown in figure 5.

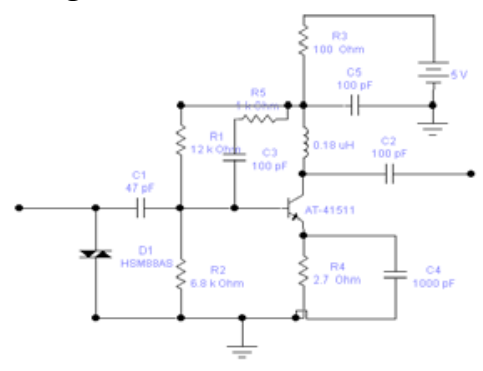

Fig.5. The high frequency amplifier circuit diagram 


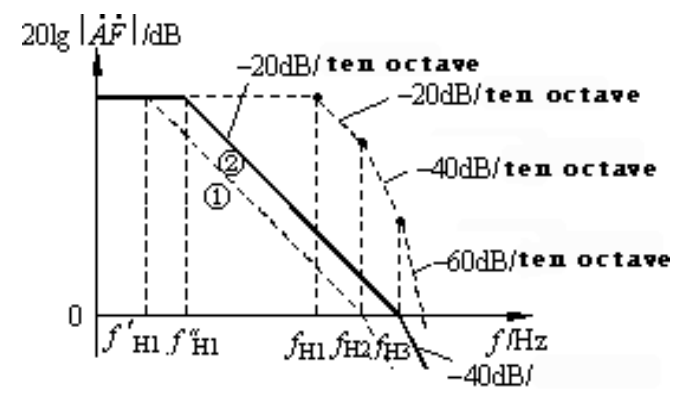

Fig.6. The amplitude frequency characteristic curve

In practice, there is a reverse transfer admittance Yre in the transistor, the output of voltage amplifier by Yre reverse effect the input of transistor, caused the change of the input current, this feedback mechanism may cause the amplifier self-excited adverse consequences[4]. If directly reducing feedback depth (such as reducing the feedback coefficient $\dot{F}$ ), it is not conductive to the other performance improvement of amplifier circuit. In order to solve this contradiction, we use the phase compensation method, namely, adding some contain reactance components of the circuit in the feedback loop, thus changing the frequency characteristics $\dot{A} \dot{F}$, destruction of self-excited oscillation conditions, such as making $f_{o}>f_{c}$ to eliminate of self-oscillation. This paper uses the $\mathrm{RC}$ delay compensation circuit, the insertion of a $\mathrm{C}$ containing capacitor circuit within a feedback loop in the phase of basic amplifying circuit, the open loop gain of lag, in order to achieve the purpose of stabilizing negative feedback amplification circuit. Using RC lag compensation can not only eliminate the self-excited oscillation, but also making the bandwidth and improving[5]. The amplitude frequency characteristics are shown by the dotted line in figure 6 .

We just choose appropriate RC parameters, make $f_{H 2}^{\prime}=f_{H 2}$, then add the RC compensation circuit, the loop gain expression is

$$
\dot{A} \dot{F}=\frac{\dot{A}_{m} \dot{F}\left(1+j \frac{f}{f_{H 2}^{\prime}}\right)}{\left(1+j \frac{f}{f_{H 1}^{\prime \prime}}\right)\left(1+j \frac{f}{f_{H 2}}\right)\left(1+j \frac{f}{f_{H 3}}\right)}=\frac{\dot{A}_{m} \dot{F}}{\left(1+j \frac{f}{f_{H 1}^{\prime \prime}}\right)\left(1+j \frac{f}{f_{H 3}}\right)}
$$

This explanation shows after joining the RC compensation circuit, there are only two turning frequency on the amplitude frequency characteristic curve of the loop gain, and if choosing $f_{H 1}^{\prime \prime}$, that section of the amplitude-frequency characteristic curve with - $20 \mathrm{~dB} /$ decade slope downward and the horizontal axis intersect at just point fH3, Here, $20 \lg |\dot{A} \dot{F}|=0 \mathrm{~dB}$, as shown in figure 5 the solid line (2), $\left(\phi_{a}+\phi_{f}\right)$ is tends to $-135^{\circ}$, so joining the negative feedback amplifier circuit of the RC lag compensation certainly not generate self-excited oscillation. The dashed line (1) in figure 5 shows the amplitude frequency characteristic using capacitive lag compensation. Obviously the upper frequency moves to the right after the RC lag compensation, that shows the bandwidth is increased.

If there is a large signal in received signals, the transistor will be likely into the supersaturated state to affect amplification performance. In this paper, we add a bidirectional diode HSM88AS in front of amplification so that the large signal loss before amplification, avoiding the influence of large signal.

In the actual circuit, through the feedback network composed of C3and R5 in figure 4 realize phase compensation. The experimental data show that the gain of the amplifier is about $30 \mathrm{dBm}$ (the input signal power of the antenna is $0 \mathrm{dBm}$, known as $1 \mathrm{MW}$ ), which is much greater than $15 \mathrm{dbm}$, so the gain is perfect.

\section{A design of the mirror frequency filter}

After a signal amplifier, it is still a narrow-band signal. because in front of this amplifier a band pass is placed, the signal through a amplifier especially the noise amplified, have a great impact on the mixer circuit back, we use the electrically tunable band pass filter. The basic principle is that using control the voltage of variable capacitor that is as in filter by the variable capacitance diode voltage, to regulate the bandwidth, filters can be translated near the center frequency. 
The performance index is:

First, At 3dB, Fp1 $=400 \mathrm{MHz}$, Fp2 $=440 \mathrm{MHz}$

Second, The bandwidth BW $=$ Fp2Fp1 $=40 \mathrm{MHz}$

Third, Gain Pg>=20dbm least.

Fourth, The impedance match $=50 \Omega$, the electric tuning can make the pass band shift $10 \mathrm{MHz}$ at

This paper designed a band-pass filter with electric tuning, as shown in figure 7. In this design we selected HVC350B model capacitance diode. The device parameters are selected according to the actual test in the circuit. The parameters are as follows:

Vrm $=15 \mathrm{~V} ; \mathrm{Rs}=0.5 \Omega$;

$\mathrm{Cd} 1=15.5 \sim 17 \mathrm{pf}(1 \mathrm{~V})$;

$\mathrm{Cd} 2=5 \sim 6 \mathrm{pF}(4 \mathrm{~V})$

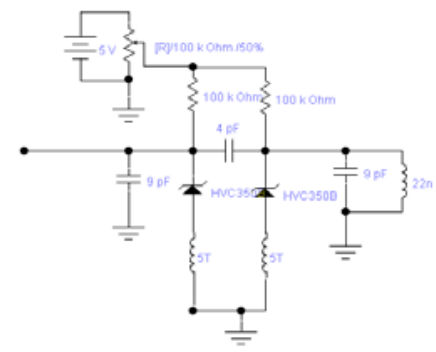

Fig.7. The circuit of mirror frequency filter

After measuring the actual circuit, the bandpass gain is about $30 \mathrm{dBm}, 3 \mathrm{~dB}$ bandwidth is about $50 \mathrm{MHz}$, basically satisfying the requirements of the circuit.

\section{Conclusion}

This paper mainly introduced a design about front-end low noise electric tuning amplifier circuit based on the receiver front-end. It discussed the design ideal and made the circuit simulation. On the basis of simulation we lap circuit, finally contrast the measured experimental data and simulation results, validating this design idea can be implemented and correctness.

\section{References}

[1] Wenhua Chen. Software experiment design of communication circuits based on ADS. submitted to Journal of Experimental Technology and Management(2011)

[2] Bo GONG, Shu-hua LI. Design and simulation to improve the low noise amplifier of communication equipment based on ADS software. submitted to Journal of Electronic Design Engineering (2010)

[3] Wei XIA, Boyu LI, Li ZHOU, Gang OU. Design of Low Noise Amplifier Based on ADS Simulation. submitted to Journal of MICROPROCESSORS (2009)

[4] Zhao NIU, Yingsong LI, Qi WANG, Xiaodong YANG. Design of wideband low noise amplifier based on ADS software simulation. submitted to Journal of Applied Science and Technology (2011)

[5] Jingchang NAN, Yongsheng FENG, Yuanan LIU. Design and Simulation of L inear Small-signal Transistor's 1. 5GHz Amplifier. submitted to Journal of Computer Simulation (2007) 